\title{
Review \\ Habitual Choline Intakes across the Childbearing Years: A Review
}

\author{
Emma Derbyshire ${ }^{1, *(\mathbb{D})}$, Rima Obeid ${ }^{2}$ and Christiane Schön ${ }^{3}(\mathbb{D}$ \\ 1 Nutritional Insight, Surrey KT17 2AA, UK \\ 2 Department of Clinical Chemistry and Laboratory Medicine, University Hospital of the Saarland, \\ D-66420 Homburg, Germany; rima.obeid@uks.eu \\ 3 BioTeSys GmbH, Schelztorstrasse 54-56, D-73728 Esslingen, Germany; c.schoen@biotesys.de \\ * Correspondence: emma@nutritional-insight.co.uk
}

check for

updates

Citation: Derbyshire, E.; Obeid, R.; Schön, C. Habitual Choline Intakes across the Childbearing Years: A Review. Nutrients 2021, 13, 4390. https://doi.org/10.3390/nu13124390

Academic Editor: David Val-Laillet

Received: 28 October 2021

Accepted: 3 December 2021

Published: 8 December 2021

Publisher's Note: MDPI stays neutral with regard to jurisdictional claims in published maps and institutional affiliations.

Copyright: (c) 2021 by the authors. Licensee MDPI, Basel, Switzerland. This article is an open access article distributed under the terms and conditions of the Creative Commons Attribution (CC BY) license (https:// creativecommons.org/licenses/by/ $4.0 /)$.

\begin{abstract}
Choline is an important nutrient during the first 1000 days post conception due to its roles in brain function. An increasing number of studies have measured choline intakes at the population level. We collated the evidence focusing on habitual choline intakes in the preconceptual, pregnancy, and lactation life stages. We conducted a review including studies published from 2004 to 2021. Twenty-six relevant publications were identified. After excluding studies with a high choline intake ( $>400 \mathrm{mg} /$ day; two studies) or low choline intake (<200 mg/day; one study), average choline intake in the remaining 23 studies ranged from $233 \mathrm{mg} /$ day to $383 \mathrm{mg} /$ day, even with the inclusion of choline from supplements. Intakes were not higher in studies among pregnant and lactating women compared with studies in nonpregnant women. To conclude, during the childbearing years and across the globe, habitual intakes of choline from foods alone and foods and supplements combined appear to be consistently lower than the estimated adequate intakes for this target group. Urgent measures are needed to (1) improve the quality of choline data in global food composition databases, (2) encourage the reporting of choline intakes in dietary surveys, (3) raise awareness about the role(s) of choline in foetal-maternal health, and (4) consider formally advocating the use of choline supplements in women planning a pregnancy, pregnant, or lactating.
\end{abstract}

Keywords: choline; essential nutrient; preconception; pregnancy; lactation; intake

\section{Introduction}

Choline is an essential nutrient involved in critical physiological functions due to its role as methyl donor and precursor of acetylcholine and phospholipids [1]. Choline requirements in pregnancy and lactation are higher than in nonpregnant women due to the rapid division of foetal cells and active transport to the foetus and infant [2]. Growing evidence implies a role for sufficient maternal choline intake in foetal growth and development [3,4]. More recently, the American Medical Association and American Academy of Paediatrics have recognised that a failure to provide choline during the first 1000 days post conception could result in lifelong deficits in brain function regardless of subsequent nutrient repletion [5-7].

Focusing on brain development, a growing body of evidence suggests that choline is an important micronutrient and methyl donor that is required for normal brain growth and development, particularly during sensitive windows of life, including the childbearing years $[1,8]$. Choline has been acknowledged as being one of six key nutrients that are important for brain development, with nutrient effects appearing to be governed by the duration, timing, and severity of a deficiency or sufficiency [9]. A previous systematic review focusing on the first 1000 days of life concluded that choline could support normal brain development, help to protect against metabolic and neural insults such as alcohol exposure, and potentially facilitate neural and cognitive function [4]. Similarly, other authors recognised that large proportions of choline-derived phospholipids, e.g., phosphatidylcholine 
and sphingomyelin, are required for cell division, growth, and myelination, which occur rapidly during foetal development [3]. The foetal origins of memory hypothesis proposes that maternal and infant dietary intakes of choline influence brain development which could permanently modulate brain function of the offspring, i.e., resulting in cognitive and memory deficits with mechanisms likely to involve DNA methylation and alterations to gene expression, as well as stem-cell proliferation and differentiation [10]. Underpinning studies in rodents further demonstrate that a higher choline intake during pregnancy facilitates cognitive function and offsets memory decline with advancing age [11].

In 2016, the European Food Safety Authority (EFSA) set an adequate intake (AI) of $400 \mathrm{mg} /$ day for all adults, $480 \mathrm{mg} /$ day for pregnant women, and $520 \mathrm{mg} /$ day for lactating women [12]. The AIs for choline according to the United States Institute of Medicine (IOM) for nonpregnant, pregnant, and lactating women are $425 \mathrm{mg} /$ day, $450 \mathrm{mg} /$ day, and $550 \mathrm{mg} /$ day, respectively [13]. The primary criterion for setting the AI for choline was the prevention of liver damage as assessed by measuring serum levels of liver enzymes, whilst a higher AI during pregnancy and lactation was justified by the extra requirements of the foetus and infant [13]. Due to inadequate data on choline intake in the population, it was not possible to derive an estimated average requirement (EAR) and recommended dietary allowance (RDA) for choline. An RDA for choline would be expected to meet the needs of $97-98 \%$ of individuals in a life stage and gender group. Since 2004, data on choline intake have become available from several populations.

Choline appears to be an under-consumed and overlooked nutrient $[14,15]$ not only in Americans [16-18] and Australasian populations [19,20], but also in Europeans [21]. In 2018, Wiedeman et al. published a review on dietary choline intake across the lifecycle which included adults and other age groups (toddlers 1-3 years, children $4-9$ years, adolescents 10-18 years, pregnancy, lactation, and the elderly $>65$ years) [22].

The present publication collates habitual choline intake data specific to the childbearing years using data from human studies published between 2004 and 2021.

\section{Materials and Methods}

\subsection{Search Strategy and Databases}

The search was conducted in the National Library of Medicine National Centre for Biotechnology Information (PubMed.gov) platform on the 8th September 2021 and updated on the 15th of November 2021 using the advanced search builder and the terms shown in Supplementary Table S1. We further searched reference lists of previous reviews and relevant studies.

The publication inclusion criteria were human studies in English language reporting habitual choline intakes from the diet in women aged $16-50$ years (as a main aim) and published between 2004 (1 January 2004) and 2021 (15 November 2021). This timeframe was specified due to the United States Department of Agriculture (USDA) Food Composition Databank being completed in 2004 [23]. The population(s) of focus were women of childbearing age (16 to 50 years) defined as those who were nonpregnant, pregnant, or lactating [24].

We planned to include studies published in English language without restriction on the country of origin. We included all study types (observational and interventional) that reported mean habitual choline intakes during the specified period. We included studies where mean habitual choline intakes were recorded without supplements (from foods only; FO) and when a combination of habitual intakes and multivitamin or mineral supplements was used (from food and supplements combined; $\mathrm{F}+\mathrm{S}$ ).

We excluded animal studies, cell culture studies, narrative reviews, case reports, and case series publications. We also omitted studies unrelated to the target population of the present review, e.g., cardiovascular or fatty liver disease. Studies with an excessively high (>400 mg/day choline) or low ( $200 \mathrm{mg} /$ day choline) intake from dietary sources alone were excluded due to intakes likely bring skewed by cultural factors or methodological limitations, e.g., small sample size or accuracy of dietary assessments. This included, for 
example, research where participants frequently ingested "menudo" (a Mexican broth using beef stomach) [25]. Studies were also excluded where the mean intake was not reported or could not be derived.

E.D. screened the titles and abstracts for relevance. The full texts of relevant articles were then reviewed, and data were extracted by E.D. and verified by R.O.

\subsection{Data Extraction}

A standardised spreadsheet was used to extract the following data: PubMed Identifier, year, first author, title, country of the participants, time period of intake assessment or pregnancy trimester, mean age, study design, sample size, how choline intake was assessed (i.e., food frequency questionnaires (FFQ), semi-quantitative food frequency questionnaire (SQ FFQ), dietary recall), and sources of choline (i.e., foods only or from foods and supplements). In addition, means and standard deviations (SDs) or medians and interquartile ranges (IQRs) of daily choline intakes (mg/day) were extracted. These were then compared against EFSA and IOM AIs for three target groups: nonpregnant, pregnant, and lactating women.

\section{Results}

\subsection{Search Results}

The search for publications was undertaken on 8 September and updated on 15 November 2021. Using the specified search terms, 91 publications were yielded. A further nine relevant studies were identified through screening reference lists. As shown in Figure 1, after screening, 74 publications were excluded, leaving 26 publications for inclusion within the main review (Tables 1-3).

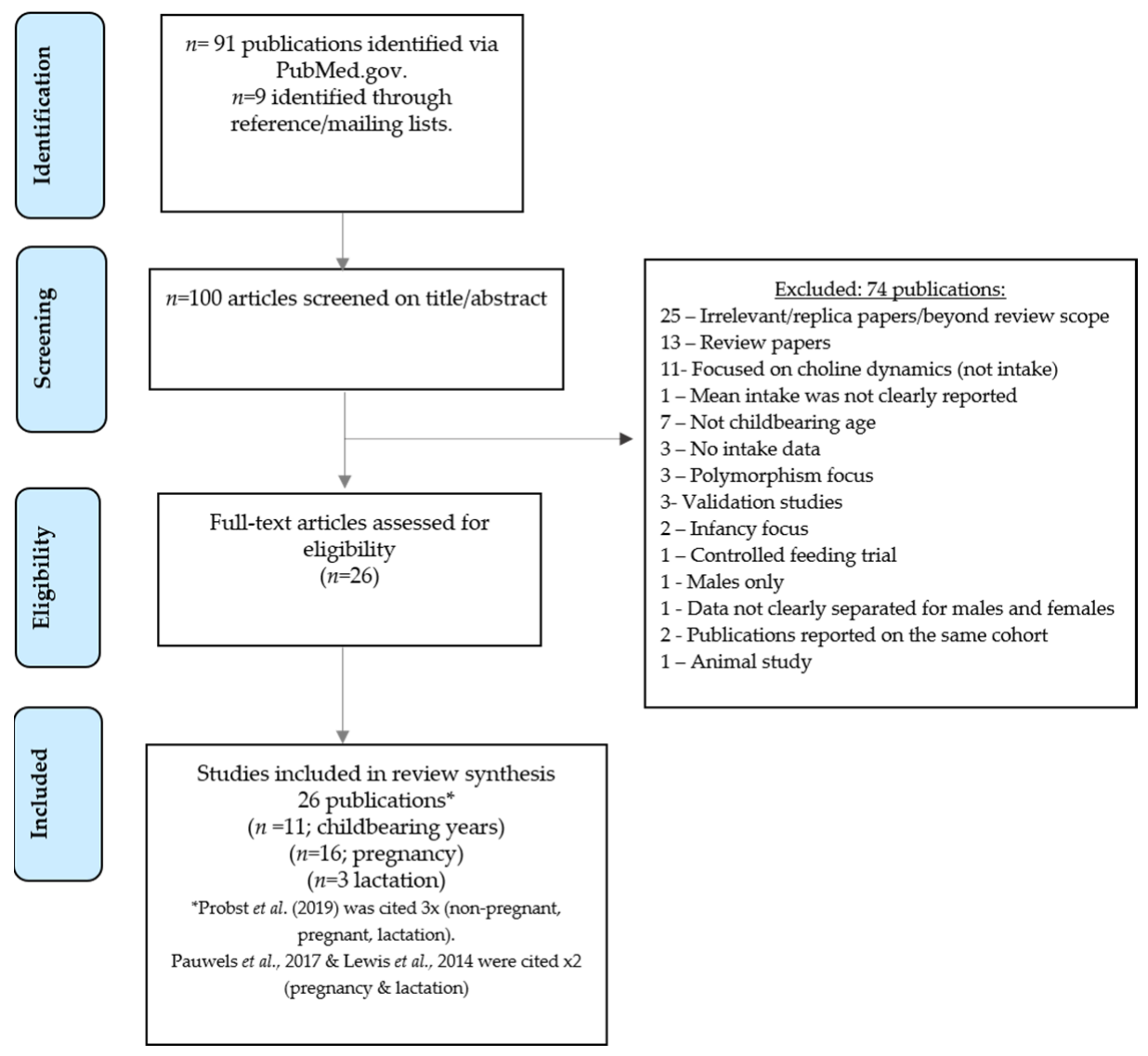

Figure 1. Study flow diagram. 
Table 1. Habitual choline intakes in nonpregnant women of childbearing age.

\begin{tabular}{|c|c|c|c|c|c|c|c|}
\hline $\begin{array}{l}\text { First Author, } \\
\text { Country }\end{array}$ & Age, Years & $\begin{array}{l}\text { Study Design, } \\
\text { Cohort Name }\end{array}$ & Sample Size & $\begin{array}{l}\text { Method and } \\
\text { Duration/Time } \\
\text { Period }\end{array}$ & $\begin{array}{l}\text { Database Used to } \\
\text { Estimate the Intake }\end{array}$ & $\begin{array}{l}\text { Source(s) } \\
\text { of Choline }\end{array}$ & $\begin{array}{l}\text { Choline Intake } \\
\text { (mg/day) }\end{array}$ \\
\hline $\begin{array}{l}\text { Petersen et al. } \\
\text { (2019), United } \\
\text { States [26] }\end{array}$ & $\begin{array}{l}\text { Range } \\
<25 \text { years } \\
(12.4 \%) \\
25-34 \text { years } \\
(68.3 \%) \\
\geq 35 \text { years } \\
(19.4 \%)\end{array}$ & $\begin{array}{l}\text { Case-control } \\
\text { study (the Slone } \\
\text { Birth Defect } \\
\text { study 1975-2014) }\end{array}$ & $\begin{array}{l}\text { Mothers of } n= \\
164 \text { NTD cases } \\
\text { (live births, } \\
\text { foetal deaths, } \\
\text { elective } \\
\text { terminations), } \\
\text { and } n=2831 \\
\text { control infants }\end{array}$ & $\begin{array}{l}\text { SQ FFQ (the } \\
\text { Willett FFQ) to } \\
\text { capture the } \\
\text { intake in the past } \\
6 \text { months }\end{array}$ & $\begin{array}{l}\text { Harvard T.H. Chan } \\
\text { School of Public } \\
\text { Health } \\
\text { Nutrition } \\
\text { Department's Food } \\
\text { Composition Table. }\end{array}$ & $\mathrm{F}+\mathrm{S}$ & $\begin{array}{l}\text { Mean (SD) }= \\
\text { Controls: } 275(68) \\
\text { Cases: } 273(67) \\
\text { Choline intake data } \\
\text { were based on } \\
\text { women with folic } \\
\text { acid intake }>400 \\
\mu \mathrm{g} / \text { day }\end{array}$ \\
\hline $\begin{array}{l}\text { Probst et al. (2019), } \\
\text { Australia [19] }\end{array}$ & $\begin{array}{l}\text { Range } \\
16-44 \text { years }\end{array}$ & $\begin{array}{l}\text { Cross-sectional } \\
\text { study based on } \\
\text { The Australian } \\
\text { Health Survey } \\
\text { 2011-13 }\end{array}$ & $n=2210$ & 2 day intake data & $\begin{array}{l}\text { Sourced studies and } \\
\text { global food } \\
\text { composition } \\
\text { databases and } \\
\text { compared these } \\
\text { with } \\
\text { data from } \\
\text { Australian foods } \\
\text { (from AUSNUT } \\
\text { 2011-13 Australian } \\
\text { Food) }\end{array}$ & FO & $\begin{array}{l}\text { Median }(\mathrm{IQR})=233 \\
(103)\end{array}$ \\
\hline $\begin{array}{l}\text { Oyen et al. (2017), } \\
\text { Norway [27] }\end{array}$ & $\begin{array}{l}\text { Range } \\
46-49 \text { years }\end{array}$ & $\begin{array}{l}\text { Cross-sectional } \\
\text { study based on } \\
\text { The Hordaland } \\
\text { Health study }\end{array}$ & $n=1600$ & $\begin{array}{l}\text { FFQ (habitual } \\
\text { diet from past } \\
\text { year) validated: } \\
169 \text { items; } \\
\text { frequency of } \\
\text { consumption } \\
\text { specified per day, } \\
\text { week, or month }\end{array}$ & $\begin{array}{l}\text { Choline intakes } \\
\text { estimated } \\
\text { according to the } \\
2008 \text { USDA choline } \\
\text { database }\end{array}$ & $\mathrm{F}+\mathrm{S}$ & $\begin{array}{l}\text { Median }(\mathrm{IQR})=255 \\
(63)\end{array}$ \\
\hline $\begin{array}{l}\text { Gao et al. (2016), } \\
\text { Canada [28] }\end{array}$ & $\begin{array}{l}\text { Mean = } 43.7 \\
\text { years }\end{array}$ & $\begin{array}{l}\text { Cross-sectional } \\
\text { CODING study } \\
\text { (Complex } \\
\text { Diseases in the } \\
\text { Newfoundland } \\
\text { population: } \\
\text { Environment } \\
\text { and Genetics) } \\
\text { study }\end{array}$ & $\begin{array}{l}n=2232-2295 \\
\text { (sample size } \\
\text { range) }\end{array}$ & $\begin{array}{l}\text { SQ FFQ (the } \\
\text { Willett FFQ) } 124 \\
\text { items; data from } \\
\text { past } 12 \text { months }\end{array}$ & $\begin{array}{l}\text { NutriBase Clinical } \\
\text { Nutrition Manager }\end{array}$ & FO & $\begin{array}{l}\text { Mean }(S D)=292 \\
(213)\end{array}$ \\
\hline $\begin{array}{l}\text { Wallace et al. } \\
\text { (2016), United } \\
\text { States [17] }\end{array}$ & $\begin{array}{l}\text { Range } \\
19-30 \text { years } \\
\text { Range } \\
31-50 \text { years }\end{array}$ & $\begin{array}{l}\text { Cross-sectional } \\
\text { study based on } \\
\text { the National } \\
\text { Health and } \\
\text { Nutrition } \\
\text { Examination } \\
\text { Survey } \\
\text { (2009-2012) }\end{array}$ & $\begin{array}{l}n=1096(19-30 \\
\text { years }) \\
n=1794(31-50 \\
\text { years })\end{array}$ & $\begin{array}{l}\text { Two } 24 \text { h dietary } \\
\text { recalls with } \\
\text { trained } \\
\text { interviewers }\end{array}$ & $\begin{array}{l}\text { Various USDA food } \\
\text { composition } \\
\text { databases }\end{array}$ & $\mathrm{F}+\mathrm{S}$ & $\begin{array}{l}\text { Mean }(S D)= \\
\text { 19-30 years: } 250 \\
(166) ; \\
\text { 31-50 years: } 278 \\
(169)\end{array}$ \\
\hline $\begin{array}{l}\text { Vennemann et al. } \\
\text { (2015), Europe [21] }\end{array}$ & $\begin{array}{l}\text { Range } \\
18-\leq 65 \text { years }\end{array}$ & $\begin{array}{l}\text { Cross-sectional } \\
\text { dietary surveys } \\
\text { in each country }\end{array}$ & $\begin{array}{l}\text { Finland } n=710 ; \\
\text { France } n=1340 ; \\
\text { Ireland } n=640 ; \\
\text { Italy } n=1245 ; \\
\text { Netherlands } \\
n=1034 ; \\
\text { Sweden } n=807 ; \\
\text { UK } n=706\end{array}$ & $\begin{array}{l}3 \text { day dietary } \\
\text { record } \\
7 \text { day dietary } \\
\text { record } \\
4 \text { day dietary } \\
\text { record } \\
3 \text { day dietary } \\
\text { record } \\
24 \text { h dietary } \\
\text { recall } \\
4 \text { day web record } \\
4 \text { day dietary } \\
\text { record }\end{array}$ & $\begin{array}{l}\text { USDA Database } \\
\text { (2013) }\end{array}$ & $\mathrm{FO}$ & $\begin{array}{l}\text { Mean (5th, 95th } \\
\text { percentiles) = } \\
\text { Finland: } 344(177, \\
\text { 578); } \\
\text { France: } 291(162, \\
\text { 440); } \\
\text { Ireland: } 318 \text { (166, } \\
\text { 485); } \\
\text { Italy: } 293(153,463) \text {; } \\
\text { Netherlands: } 334 \\
\text { (185, 543); } \\
\text { Sweden: } 374(186 \text {, } \\
\text { 631); } \\
\text { UK: } 294(145,478)\end{array}$ \\
\hline $\begin{array}{l}\text { Lavery et al. (2014), } \\
\text { Mexican } \\
\text { Americans [25] }\end{array}$ & $\begin{array}{l}\text { Range } \\
<20 \text { years } \\
(24.7 \%) \\
20-24 \text { years } \\
(34.2 \%) \\
25-29 \text { years } \\
(24 \%) \\
>30 \text { years } \\
(35 \%)\end{array}$ & $\begin{array}{l}\text { Case-control } \\
\text { study }\end{array}$ & $\begin{array}{l}\text { Mothers of } n= \\
184 \text { cases with } \\
\text { NTDs and } n= \\
225 \text { controls }\end{array}$ & $\begin{array}{l}\text { FFQ: } 98 \text { items } \\
\text { ascertaining food } \\
\text { frequency for } 3 \\
\text { months before } \\
\text { their conception } \\
\text { date to } 3 \text { months } \\
\text { postpartum }\end{array}$ & $\begin{array}{l}\text { USDA database } \\
\text { containing } 630 \text { food } \\
\text { items and six } \\
\text { choline metabolites. }\end{array}$ & $\mathrm{FO}$ & $\begin{array}{l}\text { Median }(\mathrm{IQR})= \\
\text { Controls: } 760(456) \text {; } \\
\text { Cases: } 722(689)\end{array}$ \\
\hline $\begin{array}{l}\text { Lee et al. (2010), } \\
\text { United States [29] }\end{array}$ & $\begin{array}{l}\text { Range } \\
\text { 29-86 years }\end{array}$ & $\begin{array}{l}\text { Cross=sectional } \\
\text { study in the sixth } \\
\text { examination } \\
\text { (1995-1998) of } \\
\text { the Framingham } \\
\text { Offspring Study }\end{array}$ & $n=1407$ & $\begin{array}{l}\text { FFQ, validated: } \\
130 \text { items; } \\
\text { frequency of } \\
\text { consumption } \\
\text { over the past } \\
\text { year }\end{array}$ & $\begin{array}{l}\text { Choline } \\
\text { composition data } \\
\text { values published by } \\
\text { Zeisel et al. [30] and } \\
\text { from the USDA } 2008 \\
\text { Database }\end{array}$ & $\mathrm{F}+\mathrm{S}$ & $\begin{array}{l}\text { Energy adjusted } \\
\text { mean }(\mathrm{SD})=308 \\
(56)\end{array}$ \\
\hline
\end{tabular}


Table 1. Cont.

\begin{tabular}{|c|c|c|c|c|c|c|c|}
\hline $\begin{array}{l}\text { First Author, } \\
\text { Country }\end{array}$ & Age, Years & $\begin{array}{l}\text { Study Design, } \\
\text { Cohort Name }\end{array}$ & Sample Size & $\begin{array}{l}\text { Method and } \\
\text { Duration/Time } \\
\text { Period }\end{array}$ & $\begin{array}{l}\text { Database Used to } \\
\text { Estimate the Intake }\end{array}$ & $\begin{array}{l}\text { Source(s) } \\
\text { of Choline }\end{array}$ & $\begin{array}{l}\text { Choline Intake } \\
\text { (mg/day) }\end{array}$ \\
\hline $\begin{array}{l}\text { Chiuve et al. (2007), } \\
\text { United States [31] }\end{array}$ & $\begin{array}{l}\text { Range } \\
30-55 \text { years }\end{array}$ & $\begin{array}{l}\text { Cross-sectional } \\
\text { study based on } \\
\text { the Nurses' } \\
\text { Health Study }\end{array}$ & $n=1477$ & $\begin{array}{l}\text { SQ FFQ, } \\
\text { validated: } \\
\text { undertaken } \\
\text { every 4-years }\end{array}$ & $\begin{array}{l}\text { Choline } \\
\text { composition data } \\
\text { values published by } \\
\text { Zeisel et al. [30] and } \\
\text { from the USDA } 2008 \\
\text { Database }\end{array}$ & $\mathrm{FO}$ & $\begin{array}{l}\text { Energy adjusted } \\
\text { median (range of } \\
\text { 3rd quintile) }= \\
323(311-334)\end{array}$ \\
\hline $\begin{array}{l}\text { Fischer et al. (2005), } \\
\text { United States [32] }\end{array}$ & $\begin{array}{l}\text { Range } \\
\text { 18-67 years }\end{array}$ & $\begin{array}{l}\text { Ad libitum } \\
\text { dietary intake in } \\
\text { the study centre }\end{array}$ & $n=16$ & $\begin{array}{l}\text { Choline content } \\
\text { was measured in } \\
\text { the foods and } \\
\text { compared to } \\
\text { estimates from a } \\
\text { pre-study } 3 \text { day } \\
\text { food record }\end{array}$ & $\begin{array}{l}\text { The Food Processor } \\
\text { SQL program using } \\
\text { USDA Nutrient } \\
\text { Database and Zeisel } \\
\text { et al. [30] data }\end{array}$ & FO & $\begin{array}{l}\text { Mean }(\mathrm{SD})= \\
443(88)\end{array}$ \\
\hline $\begin{array}{l}\text { Shaw et al. (2004), } \\
\text { United States [33] }\end{array}$ & $\begin{array}{l}\text { Women } \\
\text { recruited after } \\
\text { delivery, age } \\
\text { not reported }\end{array}$ & $\begin{array}{l}\text { Case-control } \\
\text { study }\end{array}$ & $\begin{array}{l}\text { Mothers of } n= \\
424 \text { cases with } \\
\text { NTDs, mothers } \\
\text { of } n=440 \\
\text { controls }\end{array}$ & $\begin{array}{l}\text { FFQ: } 100 \text { items } \\
\text { used to assess } \\
\text { frequency and } \\
\text { portion size } \\
\text { consumed } 3 \\
\text { months before } \\
\text { conception }\end{array}$ & $\begin{array}{l}\text { Used choline values } \\
\text { published by Zeisel } \\
\text { et al. [30] }\end{array}$ & $\mathrm{FO}$ & $\begin{array}{l}\text { Mean }(\mathrm{SD})= \\
\text { Controls: } 409 \text { (179); } \\
\text { Cases: } 377(176)\end{array}$ \\
\hline
\end{tabular}

FFQ, food frequency questionnaire; FO, foods only, F+S, foods and supplements; IQR; interquartile range; NTD, neural tube defects; SD, standard deviation; SQ FFQ, semi-quantitative food frequency questionnaire; USDA, United States Department of Agriculture.

Table 2. Habitual choline intakes in pregnant women.

\begin{tabular}{|c|c|c|c|c|c|c|c|}
\hline $\begin{array}{l}\text { First Author, } \\
\text { Country }\end{array}$ & Age, Years & Study Design & Sample Size & $\begin{array}{l}\text { Method and } \\
\text { Duration/ } \\
\text { Time Period }\end{array}$ & $\begin{array}{l}\text { Database Used to } \\
\text { Estimate the } \\
\text { Intake }\end{array}$ & $\begin{array}{l}\text { Source(s) } \\
\text { of Choline }\end{array}$ & $\begin{array}{l}\text { Choline Intake } \\
\text { (mg/day) }\end{array}$ \\
\hline $\begin{array}{l}\text { Fawcet et al. (2020), } \\
\text { United States [34] }\end{array}$ & Not reported. & $\begin{array}{l}\text { Prospective } \\
\text { longitudinal } \\
\text { study }\end{array}$ & $n=251$ & $\begin{array}{l}3 \text { day food records } \\
\text { completed during } \\
\text { the 1st, } 2 \text { nd, and } 3 \text { rd } \\
\text { trimester }\end{array}$ & $\begin{array}{l}\text { Nutrient Data } \\
\text { System for } \\
\text { Research }\end{array}$ & $\mathrm{FO}$ & $\begin{array}{l}\text { Mean }(\text { SD not } \\
\text { reported })= \\
281\end{array}$ \\
\hline $\begin{array}{l}\text { Moore et al. (2020), } \\
\text { United Kingdom [35] }\end{array}$ & $\begin{array}{l}\text { Mean }(\mathrm{SD})= \\
31.4(4) \text { years }\end{array}$ & $\begin{array}{l}\text { Be Healthy in } \\
\text { Pregnancy } \\
\text { (B-HIP) study, } \\
\text { baseline data of } \\
\text { a RCT }\end{array}$ & $n=232$ & $\begin{array}{l}3 \text { day weighed diet } \\
\text { record ( } 2 \text { week } \\
\text { days and } 1 \text { weekend } \\
\text { day) completed at } \\
12-17 \text { weeks } \\
\text { gestation }\end{array}$ & $\begin{array}{l}\text { Nutritionist Pro }{ }^{\mathrm{TM}} \\
\text { diet analysis } \\
\text { software }\end{array}$ & $\mathrm{F}+\mathrm{S}$ & $\begin{array}{l}\text { Median (min, } \\
\max )=338 \\
(120,1016)\end{array}$ \\
\hline $\begin{array}{l}\text { Zhu et al. (2020), } \\
\text { China [36] }\end{array}$ & $\begin{array}{l}\text { Mean }(S D)= \\
28(4) \text { years }\end{array}$ & $\begin{array}{l}\text { Case-control } \\
\text { study (SQ FFQ } \\
\text { retrospective } \\
\text { collecting } \\
\text { dietary intake } \\
\text { during } \\
\text { pregnancy) }\end{array}$ & $\begin{array}{l}\text { Mothers of } n= \\
157 \text { term controls }\end{array}$ & $\begin{array}{l}\text { SQ FFQ, validated, } \\
120 \text { food items } \\
\text { including the most } \\
\text { common foods in } \\
\text { the Chinese diet } \\
\text { conducted no later } \\
\text { than } 3 \text { days after } \\
\text { parturition }\end{array}$ & $\begin{array}{l}\text { China Food } \\
\text { Composition and } \\
\text { the USDA Food } \\
\text { Composition } \\
\text { Database }\end{array}$ & $\mathrm{FO}$ & $\begin{array}{l}\text { Energy-adjusted } \\
\text { choline intake in } \\
\text { the controls } \\
\text { Median (IQR) = } \\
255(70)\end{array}$ \\
\hline $\begin{array}{l}\text { Probst et al. (2019), } \\
\text { Australia [19] }\end{array}$ & $\begin{array}{l}\text { Range } \\
19-50 \text { years }\end{array}$ & $\begin{array}{l}\text { Cross-sectional } \\
\text { study based on } \\
\text { data from the } \\
\text { Australian } \\
\text { National } \\
\text { Nutrition and } \\
\text { Physical } \\
\text { Activity Survey } \\
2011-12\end{array}$ & $n=116$ & $\begin{array}{l}2 \text { day intake data } \\
\text { (data filtered for } \\
\text { women who were } \\
\text { pregnant at the time } \\
\text { of the survey) }\end{array}$ & $\begin{array}{l}\text { Sourced studies } \\
\text { and global food } \\
\text { composition } \\
\text { databases, } \\
\text { compared with } \\
\text { data for Australian } \\
\text { foods to create a } \\
\text { choline database }\end{array}$ & $\mathrm{FO}$ & $\begin{array}{l}\text { Median }(\mathrm{IQR})= \\
251(111)\end{array}$ \\
\hline $\begin{array}{l}\text { Bailey et al. (2019), } \\
\text { United States [16] }\end{array}$ & $\begin{array}{l}\text { Range } \\
20-40 \text { years }\end{array}$ & $\begin{array}{l}\text { Cross-sectional } \\
\text { study based on } \\
\text { data from } \\
\text { NHANES } \\
(2001-2014)\end{array}$ & $n=533$ & $\begin{array}{l}24 \text { h dietary recall } \\
\times 2 \text { (taking part in } \\
\text { the What We Eat in } \\
\text { America survey) }\end{array}$ & $\begin{array}{l}\text { USDA Food } \\
\text { Composition } \\
\text { Database } 2019\end{array}$ & FO & $\begin{array}{l}\text { Mean }(\mathrm{SD})= \\
321(231)\end{array}$ \\
\hline $\begin{array}{l}\text { Pauwels et al. (2017), } \\
\text { Belgium [37] }\end{array}$ & $\begin{array}{l}\text { Range } \\
25-41 \text { years }\end{array}$ & $\begin{array}{l}\text { A longitudinal } \\
\text { Maternal } \\
\text { Nutrition and } \\
\text { Offspring's } \\
\text { Epigenome } \\
\text { study } \\
\text { (MANOE) }\end{array}$ & $\begin{array}{l}\text { 1st trimester: } \\
n=94 \\
\text { 2nd trimester: } \\
n=85 \\
\text { 3rd trimester: } \\
n=82\end{array}$ & $\begin{array}{l}\text { FFQ, validated: } 51 \\
\text { items completed at } \\
11-13,18-22 \text {, and } \\
30-34 \text { weeks of } \\
\text { pregnancy }\end{array}$ & Not reported & $\mathrm{F}+\mathrm{S}$ & $\begin{array}{l}\text { Mean }(\mathrm{SD})= \\
\text { 1st trimester: } \\
\text { 274 (72); } \\
\text { 2nd trimester: } \\
\text { 268 (68); } \\
\text { 3rd trimester: } \\
\text { 280 (78) }\end{array}$ \\
\hline
\end{tabular}


Table 2. Cont.

\begin{tabular}{|c|c|c|c|c|c|c|c|}
\hline $\begin{array}{l}\text { First Author, } \\
\text { Country }\end{array}$ & Age, Years & Study Design & Sample Size & $\begin{array}{l}\text { Method and } \\
\text { Duration/ } \\
\text { Time Period }\end{array}$ & $\begin{array}{l}\text { Database Used to } \\
\text { Estimate the } \\
\text { Intake }\end{array}$ & $\begin{array}{l}\text { Source(s) } \\
\text { of Choline }\end{array}$ & $\begin{array}{l}\text { Choline Intake } \\
\text { (mg/day) }\end{array}$ \\
\hline $\begin{array}{l}\text { Wallace et al. (2017), } \\
\text { United States [18] }\end{array}$ & $\begin{array}{l}\text { Range } \\
13-44 \text { years }\end{array}$ & $\begin{array}{l}\text { Cross-sectional } \\
\text { study based on } \\
\text { data from the } \\
2009-2014 \text { and } \\
\text { 2005-2014 } \\
\text { NHANES } \\
\text { (2009-2014 and } \\
\text { 2005-2014) } \\
\text { datasets }\end{array}$ & $n=593$ & $\begin{array}{l}24 \mathrm{~h} \text { dietary recalls } \\
\times 2\end{array}$ & $\begin{array}{l}\text { Various USDA } \\
\text { food composition } \\
\text { databases } \\
\text { used }\end{array}$ & $\mathrm{F}+\mathrm{S}$ & $\begin{array}{l}\text { Mean (SD) = } \\
319(241)\end{array}$ \\
\hline $\begin{array}{l}\text { Groth et al. (2017), } \\
\text { United States [38] }\end{array}$ & $\begin{array}{l}\text { Range } \\
\text { 18-36 years }\end{array}$ & $\begin{array}{l}\text { Prospective } \\
\text { observational } \\
\text { study, } \\
\text { secondary } \\
\text { analysis of the } \\
\text { Limiting the } \\
\text { Phenotypic } \\
\text { Effect of } \\
\text { Pregnancy- } \\
\text { Related Weight } \\
\text { Gain }\end{array}$ & $\begin{array}{l}\text { 1st trimester: } \\
n=90 \\
\text { 2nd trimester: } \\
n=68 \\
\text { 3rd trimester: } \\
n=67\end{array}$ & $\begin{array}{l}24 \text { h dietary recalls } \\
\times 3 \text { at three } \\
\text { timepoints: early } \\
(<22 \text { weeks), mid } \\
\text { ( } 24-29 \text { weeks), and } \\
\text { late ( } 32-37 \text { weeks) } \\
\text { pregnancy. }\end{array}$ & $\begin{array}{l}\text { Nutrition Data } \\
\text { System for } \\
\text { Research software } \\
2009\end{array}$ & FO & $\begin{array}{l}\text { Mean }(\mathrm{SD})= \\
1 \mathrm{~s}^{t} \text { trimester: } \\
318(68) ; \\
\text { 2nd trimester: } \\
\text { 289 (28); } \\
\text { 3rd trimester: } \\
\text { 306 (28) }\end{array}$ \\
\hline $\begin{array}{l}\text { Masih et al. (2015), } \\
\text { Canada [39] }\end{array}$ & $\begin{array}{l}\text { Mean }(\mathrm{SD})= \\
32(5) \text { years }\end{array}$ & $\begin{array}{l}\text { Prospective } \\
\text { observational } \\
\text { study, the } \\
\text { Prenatal Folic } \\
\text { Acid Exposure } \\
\text { on DNA } \\
\text { Methylation in } \\
\text { the newborn } \\
\text { infant study }\end{array}$ & $n=290$ & $\begin{array}{l}\text { SQ FFQ, validated: } \\
110 \text { items; recall of } \\
\text { habitual intakes } \\
\text { between } 0-16 \text { and } \\
23-27 \text { weeks } \\
\text { gestation }\end{array}$ & $\begin{array}{l}\text { Nutrition Quest } \\
\text { used the nutrient } \\
\text { composition data } \\
\text { primarily from } \\
\text { version } 1.0 \text { of the } \\
\text { USDA Food and } \\
\text { Nutrient Database }\end{array}$ & $\mathrm{F}+\mathrm{S}$ & $\begin{array}{l}\text { Mean }(\mathrm{SD})= \\
\text { 1st trimester: } \\
\text { 306 (127); } \\
\text { 3rd trimester: } \\
302(122)\end{array}$ \\
\hline $\begin{array}{l}\text { Goon et al. (2014), } \\
\text { Bangladesh [40] }\end{array}$ & $\begin{array}{l}\text { Range } \\
21-25 \text { years }\end{array}$ & $\begin{array}{l}\text { Cross-sectional } \\
\text { study }\end{array}$ & $n=103$ & $\begin{array}{l}24 \text { h dietary recall in } \\
\text { the } 7 \text { th, } 8 \text { th, or } 9 \text { th } \\
\text { months of } \\
\text { pregnancy }\end{array}$ & $\begin{array}{l}\text { USDA Food } \\
\text { Composition } \\
\text { database }\end{array}$ & FO & $\begin{array}{l}\text { Mean }(\mathrm{SD})= \\
190(98)\end{array}$ \\
\hline $\begin{array}{l}\text { Lewis et al. (2014), } \\
\text { Canada [41] }\end{array}$ & $\begin{array}{l}\text { Range } \\
17-30 \text { years } \\
(45.8 \%) \\
31-45 \text { years } \\
(54.2 \%)\end{array}$ & $\begin{array}{l}\text { Prospective } \\
\text { cohort study, } \\
\text { the Alberta } \\
\text { Pregnancy } \\
\text { Outcomes and } \\
\text { Nutrition } \\
\text { (APrON) cohort } \\
\text { study }\end{array}$ & $\begin{array}{l}\text { 1st trimester: } \\
n=123 \\
\text { 2nd trimester: } \\
n=562 \\
\text { 3rd trimester: } \\
n=493\end{array}$ & $\begin{array}{l}24 \mathrm{~h} \text { dietary recall } \\
\text { using the } \\
\text { multiple-pass } \\
\text { method in each } \\
\text { trimester }\end{array}$ & $\begin{array}{l}\text { The Alberta } \\
\text { database used the } \\
\text { USDA } \\
\text { Database for the } \\
\text { Choline Content of } \\
\text { Common Foods, } \\
\text { Release } 2 \text { ( } 634 \\
\text { foods) }\end{array}$ & $\mathrm{F}+\mathrm{S}$ & $\begin{array}{l}\text { Mean }(\mathrm{SD})= \\
\text { 1st trimester: } \\
\text { 340 (148); } \\
\text { 2nd trimester: } \\
\text { 349 (154); } \\
\text { 3rd trimester: } \\
\text { 353 (144) }\end{array}$ \\
\hline $\begin{array}{l}\text { Mygind et al. (2013), } \\
\text { New Zealand [20] }\end{array}$ & $\begin{array}{l}\text { Range 18-40 } \\
\text { years }\end{array}$ & $\begin{array}{l}\text { Baseline part of } \\
\text { dietary data } \\
\text { collection for a } \\
\text { folate } \\
\text { intervention } \\
\text { study }\end{array}$ & $n=125$ & $\begin{array}{l}3 \text { day weighed food } \\
\text { record Two } \\
\text { weekdays and one } \\
\text { weekend day }\end{array}$ & $\begin{array}{l}\text { USDA Choline } \\
\text { Content of } \\
\text { Common Foods, } \\
\text { Release 2, } 2008\end{array}$ & $\mathrm{FO}$ & $\begin{array}{l}\text { Median }(\mathrm{IQR})= \\
310(87)\end{array}$ \\
\hline $\begin{array}{l}\text { Wu et al. (2012), } \\
\text { Canada [42] }\end{array}$ & Not reported & $\begin{array}{l}\text { Prospective } \\
\text { study }\end{array}$ & $n=154$ & $\begin{array}{l}\text { FFQ, women } \\
\text { enrolled from } 16 \\
\text { weeks gestation }\end{array}$ & $\begin{array}{l}\text { USDA Choline } \\
\text { Content of } \\
\text { Common Foods, } \\
\text { Release 2, } 2008\end{array}$ & $\mathrm{FO}$ & $\begin{array}{l}\text { Mean }(S D)= \\
383(99)\end{array}$ \\
\hline $\begin{array}{l}\text { Villamor et al. (2012), } \\
\text { United States [43] }\end{array}$ & $\begin{array}{l}\text { Mean }(S D)= \\
33(5) \text { years }\end{array}$ & $\begin{array}{l}\text { Project Viva } \\
\text { longitudinal } \\
\text { study }\end{array}$ & $\begin{array}{l}\text { 1st trimester: } \\
n=1148 \\
\text { 2nd trimester: } \\
n=1083\end{array}$ & $\begin{array}{l}\text { FFQ in the first and } \\
\text { second trimester } \\
\text { (26-28 weeks } \\
\text { gestation) }\end{array}$ & $\begin{array}{l}\text { Harvard nutrient } \\
\text { composition } \\
\text { database }\end{array}$ & $\mathrm{F}+\mathrm{S}$ & $\begin{array}{l}\text { Mean }(\mathrm{SD})= \\
\text { 1st trimester: } \\
332(63) ; \\
\text { 2nd trimester: } \\
325(64)\end{array}$ \\
\hline $\begin{array}{l}\text { EFSA }(2016), \\
\text { Latvia }[12,44]\end{array}$ & $\begin{array}{l}\text { Range } \\
15-45 \text { years }\end{array}$ & $\begin{array}{l}\text { Food } \\
\text { Consumption } \\
\text { data from the } \\
\text { EFSA European } \\
\text { Comprehensive } \\
\text { Food } \\
\text { Consumption } \\
\text { Database }\end{array}$ & $n=990$ & $\begin{array}{l}24 \mathrm{~h} \text { dietary recall in } \\
\text { pregnancy }\end{array}$ & Not reported & FO & $\begin{array}{l}\text { Pregnant } \\
\text { adolescents: } \\
\text { Mean = 336; } \\
\text { Pregnant } \\
\text { women: } \\
\text { Mean (5th, 95th } \\
\text { percentiles) = 356 } \\
(200,592)\end{array}$ \\
\hline $\begin{array}{l}\text { Gossell-Williams et al. } \\
\text { (2005), Jamaica [45] }\end{array}$ & $\begin{array}{l}\text { Range } \\
18-32 \text { years }\end{array}$ & $\begin{array}{l}\text { Observational } \\
\text { study }\end{array}$ & $n=16$ & $\begin{array}{l}\text { FFQ (Jamaican } \\
\text { foods) recruited at } \\
10-15 \text { weeks } \\
\text { gestation }\end{array}$ & $\begin{array}{l}\text { USDA Food } \\
\text { Composition } \\
\text { database }\end{array}$ & FO & $\begin{array}{l}\text { Mean }(S D)= \\
279(116)\end{array}$ \\
\hline
\end{tabular}

EFSA, European Food Safety Authority; FFQ, food frequency questionnaire; FO, foods only; F+S, foods and supplements; IQR; interquartile range; NHANES, National Health and Nutrition Examination Survey; RCT, randomised controlled trial; SD, standard deviation; SQ FFQ, semi-quantitative food frequency questionnaire; USDA, United States Department of Agriculture. 
Table 3. Habitual choline intakes in lactating women.

\begin{tabular}{|c|c|c|c|c|c|c|c|}
\hline $\begin{array}{l}\text { First Author, } \\
\text { Country }\end{array}$ & Age, Years & Study Design & Sample Size & $\begin{array}{l}\text { Method and } \\
\text { Duration/Time } \\
\text { Period }\end{array}$ & $\begin{array}{l}\text { Database Used to } \\
\text { Estimate the Intake }\end{array}$ & $\begin{array}{l}\text { Source(s) } \\
\text { of Choline }\end{array}$ & $\begin{array}{l}\text { Choline Intake } \\
\text { (mg/day) }\end{array}$ \\
\hline $\begin{array}{l}\text { Probst et al. (2019), } \\
\text { Australia [19] }\end{array}$ & $\begin{array}{l}\text { Range } \\
19-50 \text { years }\end{array}$ & $\begin{array}{l}\text { Cross-sectional } \\
\text { study based on data } \\
\text { from the Australian } \\
\text { National Nutrition } \\
\text { and Physical } \\
\text { Activity Survey } \\
2011-12\end{array}$ & $n=110$ & $\begin{array}{l}2 \text { day intake data } \\
\text { analysed from } \\
\text { 'lactating } \\
\text { mothers' }\end{array}$ & $\begin{array}{l}\text { Sourced studies and } \\
\text { global food composition } \\
\text { databases, compared } \\
\text { these with data for } \\
\text { Australian foods to } \\
\text { create a choline } \\
\text { database }\end{array}$ & $\mathrm{FO}$ & $\begin{array}{l}\text { Median }(\mathrm{IQR})= \\
257(100)\end{array}$ \\
\hline $\begin{array}{l}\text { Pauwels et al. (2017), } \\
\text { Belgium [37] }\end{array}$ & $\begin{array}{l}\text { Range } \\
\text { 25-41 years }\end{array}$ & $\begin{array}{l}\text { The Maternal and } \\
\text { Offspring's } \\
\text { Epigenome } \\
\text { prospective, } \\
\text { observational cohort } \\
\text { study }\end{array}$ & $\begin{array}{l}6-8 \text { weeks PP: } \\
n=79 ; \\
6 \text { months PP: } \\
n=60\end{array}$ & $\begin{array}{l}\text { FFQ, validated } \\
51 \text {-items } \\
\text { conducted at } 6-8 \\
\text { weeks and } 6 \\
\text { months } \\
\text { postpartum }\end{array}$ & Not reported & $\mathrm{F}+\mathrm{S}$ & $\begin{array}{l}\text { Mean }(\mathrm{SD})= \\
6-8 \text { weeks PP: } \\
278(76) ; \\
6 \text { months PP: } \\
268(60)\end{array}$ \\
\hline $\begin{array}{l}\text { Lewis et al. (2014), } \\
\text { Canada [41] }\end{array}$ & $\begin{array}{l}\text { Range } \\
17-30 \text { years } \\
(45.8 \%) \\
31-45 \text { years } \\
(54.2 \%)\end{array}$ & $\begin{array}{l}\text { Alberta Pregnancy } \\
\text { Outcomes and } \\
\text { Nutrition (APrON) } \\
\text { cohort study }\end{array}$ & $n=488$ & $\begin{array}{l}24 \text { h dietary } \\
\text { recall using the } \\
\text { multiple-pass } \\
\text { method }\end{array}$ & $\begin{array}{l}\text { The Alberta database } \\
\text { was developed using } \\
\text { the USDA } \\
\text { Database for the } \\
\text { Choline Content of } \\
\text { Common Foods, } \\
\text { Release } 2 \text { ( } 634 \text { foods) }\end{array}$ & $\mathrm{F}+\mathrm{S}$ & $\begin{array}{l}\text { Mean }(S D)= \\
346(151)\end{array}$ \\
\hline
\end{tabular}

FFQ, food frequency questionnaire; FO, foods only, F+S, foods and supplements; IQR; interquartile range; PP, postpartum; SD, standard deviation; USDA, United States Department of Agriculture.

\subsection{Childbearing Age}

Eleven studies measured choline intakes amongst women of childbearing age $[17,19,21,25-29,31-33]$. Seven studies recorded choline intakes derived from food sources, $[19,21,25,28,31-33]$. Highest median choline intake $(760 \mathrm{mg} /$ day in cases and $722 \mathrm{mg} /$ day in controls) was reported in women from the Texas-Mexico border, possibly due to menudo (a traditional Mexican broth made with beef stomach) [25]. After excluding this study, choline intakes in the remaining seven publications ranged from 244 to $443 \mathrm{mg}$ /day from food sources [19,21,28,31-33].

The remaining four publications derived choline intakes from food and supplemental sources $[17,26,27,29]$. When supplements were included, choline intakes ranged from $250 \mathrm{mg} /$ day in research conducted by Wallace et al. (2016) [17] to $308 \mathrm{mg} /$ day in the cross-sectional study undertaken by Lee et al. (2010) [29].

Focusing on European data, Vennemann et al. (2015) [21] estimated choline intake from food sources in females (age 18-65 years) from Finland, France, Ireland, Italy, Netherlands, Sweden, and the United Kingdom. Mean choline intakes from food sources in this European-wide population study ranged from $291 \mathrm{mg} /$ day in France to $374 \mathrm{mg} /$ day in Sweden. Similar choline intake estimates were reported in women from countries outside the European region. American studies amongst women of childbearing age have reported choline intakes ranging between 250 and $443 \mathrm{mg} /$ day [17,26,29,31-33].

In Australia, mean choline intakes for women of childbearing age (16-44 years) were $244 \mathrm{mg} /$ day (95\% CI 246-255) from food sources [19]. Only 4.75\% women achieved choline intakes equivalent to or higher than the country's AI of $425 \mathrm{mg} /$ day [19].

\subsection{Pregnancy}

Sixteen studies recorded choline intakes during pregnancy [16,18-20,34-45]. Seven documented choline intakes in the first trimester [35,37-39,41,43,45], along with five in the second trimester [37,38,41-43], five in the third trimester [37-41], and six in pregnancy overall $[16,18-20,34,44]$. Across the trimesters, changes in choline intake were generally very small. Two studies reported a $12-13 \mathrm{mg} /$ day increase in choline intake between the first and thirst trimesters [37,41]. Two publications recorded a decline in mean choline intakes between the first and third trimesters $(-4$ and $-12 \mathrm{mg} /$ day) $[38,39]$.

Mean choline intake estimates for pregnant adults from the United States ranged from $281 \mathrm{mg} /$ day to $332 \mathrm{mg} /$ day $[16,18,34,38,43]$. Intakes were similar in pregnant Canadians with mean intake estimates ranging between $306 \mathrm{mg} /$ day and $383 \mathrm{mg} /$ day [39,41,42,46]. 
Moore et al. (2020) [35] reported a median choline intake (from foods and supplements) of $338 \mathrm{mg} /$ day in UK pregnant women. Median dietary choline intake in Australian pregnant females (aged 19 to 50 years) was $251 \mathrm{mg} /$ day [19]. Similar intakes were reported in human studies conducted in Belgium (mean intakes: $274 \mathrm{mg} /$ day (first trimester)-280 mg/day (third trimester)) [37], China (median $255 \mathrm{mg}$ /day) [36], Jamaica (mean $279 \mathrm{mg} /$ day) [45], and marginally higher in New Zealand (median $310 \mathrm{mg} /$ day) [20].

Mean choline intake in Latvian pregnant adolescents was $336 \mathrm{mg} /$ day [44]. Lowest choline intakes were reported by young women (21-25 years) in their third trimester in Bangladesh (190 mg/day) [40].

The inclusion of dietary supplements did not substantially increase choline intakes. When derived from foods only, mean choline intake estimates for pregnant adults ranged from 190-383 mg/day [19,20,34,36,38,40,42]. In studies when estimates included choline from foods and supplements, mean intakes ranged from 268 to $353 \mathrm{mg} /$ day and, thus, did not change substantially $[16,18,35,37,39,41,43,46]$.

\subsection{Lactation}

Three studies recorded the mothers mean choline intakes during lactation [19,37,41]. Postpartum intakes of choline were recorded in research conducted in Belgium [37], Canada [41], and Australia [19]. Lowest intakes were reported in Australia (median $257 \mathrm{mg} /$ day) [19], and highest intakes were reported in Canada (mean 346 mg/day) [41].

\section{Discussion}

Choline is a critical nutrient during pregnancy and lactation because it plays a central role in foetal and child development $[1,3,4,8]$. Therefore, we investigated the current evidence on whether women of childbearing age, pregnant women, or lactating women are achieving sufficient choline through their diet or a combination of diet and supplements. The majority of studies we identified among these groups of women (24 studies) reported average choline intakes below the AIs as recommended by the EFSA and IOM (Supplemental Figures S1-S3). Two studies reported mean choline intake estimates over $400 \mathrm{mg} /$ day [25,32]. These were conducted in Mexican American populations or the United States. Lavery et al. (2014) reported the highest average choline intakes $(818 \mathrm{mg} /$ day in controls), which was attributed to the consumption of "menudo" - a traditional Mexican soup made into a broth using beef stomach [25]. The remaining study by Fischer et al. (2005) reported a mean intake of $443 \mathrm{mg} /$ day in nonpregnant women $(n=16)$, only just exceeding the AI for nonpregnant women ( $400 \mathrm{mg} /$ day) but below the EFSA AI for pregnancy (i.e., $480 \mathrm{mg} /$ day) [32,33]. With the exception of these two studies, choline intakes from studies in the US were similar to those reported in European studies. Five percent of the women had choline intake above $631 \mathrm{mg} /$ day in Sweden, above $578 \mathrm{mg} /$ day in Finland, and above $543 \mathrm{mg} /$ day in the Netherlands [21].

Lowest intakes were reported during pregnancy in Bangladesh (190 mg/day) by Goon and Dey (2014), who described that the retrospective $24 \mathrm{~h}$ dietary recall may have been subject to limitations [40]. The average intakes from the remaining studies ranged from $233 \mathrm{mg} /$ day to $383 \mathrm{mg} /$ day. The reported studies show large variations in average choline intakes between different countries and studies (or ethnic groups) within one country. Moreover, European studies suggest the presence of a south to north gradient, with intakes likely to be slightly higher in Nordic countries than in southern Europe. Choline intake recommendations on a population level rely mainly on intake data from representative studies, especially as there are no optimal biomarkers to measure choline status at present.

Overarching results clearly demonstrate that most women of childbearing age across the globe are not likely to meet the current AIs for choline. Moreover, the studies have shown that average choline intakes are not higher in pregnant and lactating women compared to nonpregnant women, suggesting the urgent need to stress the recommendations of achieving sufficient choline intake in women. Higher maternal choline intake has been associated with a lower odds ratio of neural tube defects [33], and experimental choline 
deficiency is associated with brain malformation [47,48]; thus, it can be argued that choline should be added to prenatal supplements.

The gap in choline intake is approximately $70-100 \mathrm{mg} /$ day, which needs to be replaced via prenatal supplements. There are several supplemental forms of choline such as choline bitartrate, choline chloride, and phosphatidylcholine. There is currently no sufficient evidence that either one of these forms is preferable. However, the disadvantage of phosphatidylcholine is that a large dose is required to achieve the required choline intake, which could be a limitation when combining phosphatidylcholine with other vitamins, as two capsules may need to be supplemented.

In terms of dietary trends, whilst plant source foods can provide some choline [3], it is well recognised that animal-derived foods such as meat, eggs, milk, and fish tend to provide more choline per unit weight than plant-based foods such as fruit, vegetables, and grains [30,49-51]. The global trend to reduce animal-source foods implies that many women of childbearing age could have further decrements in choline intakes $[14,15]$. For example, Lecorguille et al. (2020) observed that the "vegetarian tendency" dietary pattern was associated with lower intake coefficients for choline, vitamin B12, and methionine [52]. Metabolomic research showed that an omnivorous breakfast resulted in higher choline concentrations in serum compared with lacto-/ovo-vegetarian and vegan options [53]. A cross-sectional evaluation of 74 US lactating women showed large between-individual variations in total water-soluble choline levels in samples of breast milk (range between 4 and $301 \mathrm{mg} / \mathrm{L}$ ). While total water-soluble choline forms in breast milk did not significantly differ with maternal diet, mean choline derived from glycerophosphocholine was $20 \%$ higher, and mean choline from phosphocholine was $12 \%$ lower in vegan mothers compared to nonvegetarian mothers [54]. Another study showed that concentrations of water-soluble choline forms in mature milk did not significantly differ between lactating women in Canada and Cambodia despite likely lower choline intakes in Cambodian women compared to Canadian women [55]. Altogether, these studies are difficult to compare due to variations in intakes and sources of choline in maternal diet, as well as due to analytical methods of breastmilk choline without taking fat-soluble choline derivatives into account.

Findings show that it is necessary to increase knowledge about choline during these important life stages. Whilst diets may improve somewhat in pregnancy, dietary planning tends to become more challenging postpartum [56,57]. Swedish research showed that women's diet quality declined postpartum, mainly due to increased intakes of discretionary foods [56]. Similarly, amongst Australian first-time mothers, only $8.6 \%$ met guidelines for combined fruit and vegetable intake, indicating a gap in the distribution of healthy eating advice after birth [57]. Subsequently, particular attention should be paid to these life stages. These findings imply that women are not fully adopting basic healthy eating guidelines; thus, much will need to be done to raise awareness about the role(s) of specific nutrients such as choline.

The present study identified gaps in knowledge, sources of heterogeneity, and possible topics to be improved in future studies. The limitations of the present study are its inclusion of only English literature, the search being limited to the PubMed platform and reference searches, and the lack of a plan to perform a systematic search combined with quantitative data analysis.

\section{Limitations in the Evidence and Gaps in Knowledge Surrounding Choline Intake}

Most studies reported that they used 'validated' methods to determine choline intakes. Two publications, for example, used the Willett SQ FFQ [26,28,58]. However, it was unclear whether these were fully validated for their accuracy in relation to obtaining suitable energy intakes and/or preventing under-reporting. In Cape Town, one quantitative FFQ appears to have been developed and validated to determine choline intakes in pregnant women [59]. This specific tool comprised 10 food groups (beef, lamb, chicken, processed meat, fish, eggs, vegetables, fruit, dairy, and other items) and demonstrated reliability and validity, thus having potential to be used in other communities where choline inadequacy may exist [59]. 
Weighed-intake food records could also be used as these tend to provide precise estimates of portion consumed, with evolving technologies helping to ease recording burdens placed on participants [60]. Levels of under- and over-reporting also need to be accounted for, using methods such as the Goldberg equation [61]. Plasma free choline can also be used as a biomarker to assess choline status but can be highly variable [62]. The majority of studies used the USDA database to assess data on the choline composition of foods or software programs into which this information was integrated. Several publications also used data from Zeisel et al. (2003), who published values in relation to the concentrations of choline compounds found in foods [30]. It is worth noting that the USDA database does not yet contain food composition values for all foods. Given the changing dynamics to current food markets (moving away from animal-derived towards plant-derived proteins), there is a need to urgently add to and update such databases.

Updated studies are needed using rigorous methodologies to estimate the intake of choline and choline-containing phospholipids from different foods and link the intake and bioavailability to the requirements. Future studies also need to reduce variability in methods used to estimate choline intake and establish country-specific food tables that contain choline and choline-related compounds. Cooking methods have been found to reduce the relative percentage from free choline whilst increasing the contribution of phosphatidylcholine to total choline for most pulses [49]. Cooking may cause up to $\pm 30 \%$ variability of choline content per serving compared with US Department of Agriculture database figures [49]. Mincing raw vegetables can also reduce phosphatidylcholine content by activating phospholipase D, thus releasing free choline and phosphatidic acid [30]. Moreover, variations in gut microbiome, polymorphisms, and other metabolic factors could influence the bioavailability and turnover of choline in the body [63,64]. In addition, lifestyle factors such as adherence to a vegan/vegetarian diet are associated with consuming different sources and forms of dietary choline.

In addition, several studies noted that supplements were included but did not provide a breakdown of intake data from dietary sources per se and dietary sources and supplements separately. When supplements were used, studies rarely specified 'how much' choline was provided from supplements. As separate choline supplements are not yet widely available or known about, and as choline is not yet presently added to most multivitamin and mineral preparations, this was potentially overlooked $[5,14]$. However, it would be useful to segregate and account for this in future studies.

On a final point, the reference intake of choline increases during pregnancy and lactation compared to that in nonpregnant women. An RDA for choline has not yet been established due to a lack of population-representative intake data. Thus, population intake data, especially in vulnerable groups may help in defining an RDA for choline. Our study mapping studies on choline intake throughout the childbearing age is a first step towards establishment of average population intakes of choline.

\section{Final Evaluation and Conclusions}

In summary, average choline intakes in women from different countries across the globe appeared to be lower than IOM or EFSA guidance, and intake values were generally not higher in studies among pregnant and lactating women compared to studies among nonpregnant women, thus indicating that a considerable proportion of women are not meeting the AI. This is concerning given the growing body of evidence relating choline to foetal and child development $[1,4,5,8,65]$. Taken collectively, a number of urgent measures are needed. This includes (1) adding and improving data quality in food composition tables to facilitate the collection of information on choline intake from different populations, (2) integrating and reporting choline intakes in dietary survey across the globe, (3) updating health policies and embedding choline within these to raise awareness about its role(s) in foetal-maternal health, and (4) formally recommending that women planning a pregnancy, as well as pregnant or lactating women, should supplement their diets with choline. 
Supplementary Materials: The following are available online at https:/ / www.mdpi.com/article/10 .3390/nu13124390/s1: Figure S1. Mean/median choline intakes (mg/day) reported in studies among nonpregnant women; Figure S2. Mean/median choline intakes (mg/day) reported in studies among pregnant women; Figure S3. Mean/median choline intakes (mg/day) reported in studies among lactating women; Table S1. Search key terminologies.

Author Contributions: E.D. conducted the literature search, screening, data extraction, and data analyses; R.O. verified data extraction and provided input into the discussion. E.D., R.O. and C.S. reviewed the final draft. All authors have read and agreed to the published version of the manuscript.

Funding: The research was sponsored by P\&G Health Germany GmbH.

Conflicts of Interest: E.D. was employed by the company Nutritional Insight, Ltd. and acted as an independent nutrition consultant in the research and writing of this article. E.D. received a consulting honorarium from $P \& G$ and has previously participated in advisory boards on choline for Procter 7 Gamble Health, Germany GmbH. R.O. has received consulting and speaker honoraria from P\&G. C.S. declares no conflict of interest.

\section{References}

1. Blusztajn, J.K.; Slack, B.E.; Mellott, T.J. Neuroprotective Actions of Dietary Choline. Nutrients 2017, 9, 815. [CrossRef]

2. Radziejewska, A.; Chmurzynska, A. Folate and choline absorption and uptake: Their role in fetal development. Biochimie 2019, 158, 10-19. [CrossRef] [PubMed]

3. Korsmo, H.W.; Jiang, X.; Caudill, M.A. Choline: Exploring the Growing Science on Its Benefits for Moms and Babies. Nutrients 2019, 11, 1823. [CrossRef] [PubMed]

4. Derbyshire, E.; Obeid, R. Choline, Neurological Development and Brain Function: A Systematic Review Focusing on the First 1000 Days. Nutrients 2020, 12, 1731. [CrossRef]

5. Wallace, T.C.; Blusztajn, J.K.; Caudill, M.A.; Klatt, K.C.; Zeisel, S.H. Choline: The Neurocognitive Essential Nutrient of Interest to Obstetricians and Gynecologists. J. Diet Suppl. 2020, 17, 733-752. [CrossRef] [PubMed]

6. AAP. American Academy of Paediatrics. Food for Thought: AAP Aims to Ensure Kids Get Key Nutrients for Brain Development. 2018. Available online: https://www.aap.org/en-us/about-the-aap/aap-press-room/Pages/Food-for-Thought-AAP-Aimsto-Ensure-Kids-Get-Key-Nutrients-for-Brain-Development.aspx (accessed on 12 April 2021).

7. Berg, S. American Medical Association. AMA backs Global Health Experts in Calling Infertility a Disease. 2017. Available online: https:/ / www.ama-assn.org/delivering-care/public-health/ama-backs-global-health-experts-calling-infertility-disease (accessed on 10 April 2021).

8. Bekdash, R.A. Neuroprotective Effects of Choline and Other Methyl Donors. Nutrients 2019, 11, 2995. [CrossRef] [PubMed]

9. Cheatham, C.L. Nutritional Factors in Fetal and Infant Brain Development. Ann. Nutr. Metab. 2019, 75 (Suppl. 1), $20-32$. [CrossRef] [PubMed]

10. Zeisel, S.H. The fetal origins of memory: The role of dietary choline in optimal brain development. J. Pediatr. 2006, 149, 131-136. [CrossRef]

11. Blusztajn, J.K.; Mellott, T.J. Choline nutrition programs brain development via DNA and histone methylation. Cent. Nerv. Syst. Agents Med. Chem. 2012, 12, 82-94. [CrossRef] [PubMed]

12. EFSA. Dietary Reference Values for Choline. EFSA Panel on Dietetic Products, Nutrition and Allergies (NDA). EFSA J. 2016, 14, 4484.

13. IoM. Institute of Medicine. Dietary Reference Intakes for Thiamin, Riboflavin, Niacin, Vitamin B6, Folate, Vitamin B12, Pantothenic Acid, Biotin and Choline; National Academy Press: Washington, DC, USA, 1998.

14. Wallace, T.C.; Blusztajn, J.K.; Caudill, M.A.; Klatt, K.C.; Natker, E.; Zeisel, S.H.; Zelman, K.M. Choline: The Underconsumed and Underappreciated Essential Nutrient. Nutr. Today 2018, 53, 240-253. [CrossRef] [PubMed]

15. Derbyshire, E. Could we be overlooking a potential choline crisis in the United Kingdom? BMJ Nutr. Prev. Health 2019, 2, 86-89. [CrossRef] [PubMed]

16. Bailey, R.L.; Pac, S.G.; Fulgoni, V.L., 3rd; Reidy, K.C.; Catalano, P.M. Estimation of Total Usual Dietary Intakes of Pregnant Women in the United States. JAMA Netw. Open 2019, 2, e195967. [CrossRef] [PubMed]

17. Wallace, T.C.; Fulgoni, V.L., 3rd. Assessment of Total Choline Intakes in the United States. J. Am. Coll. Nutr. 2016, 35, 108-112. [CrossRef] [PubMed]

18. Wallace, T.C.; Fulgoni, V.L. Usual Choline Intakes Are Associated with Egg and Protein Food Consumption in the United States. Nutrients 2017, 9, 839. [CrossRef] [PubMed]

19. Probst, Y.; Guan, V.; Neale, E. Development of a Choline Database to Estimate Australian Population Intakes. Nutrients 2019, 11, 913. [CrossRef]

20. Mygind, V.L.; Evans, S.E.; Peddie, M.C.; Miller, J.C.; Houghton, L.A. Estimation of usual intake and food sources of choline and betaine in New Zealand reproductive age women. Asia Pac. J. Clin. Nutr. 2013, 22, 319-324. [CrossRef] 
21. Vennemann, F.B.; Ioannidou, S.; Valsta, L.M.; Dumas, C.; Ocke, M.C.; Mensink, G.B.; Lindtner, O.; Virtanen, S.M.; Tlustos, C.; D'Addezio, L.; et al. Dietary intake and food sources of choline in European populations. Br. J. Nutr. 2015, 114, $2046-2055$. [CrossRef]

22. Wiedeman, A.M.; Barr, S.I.; Green, T.J.; Xu, Z.; Innis, S.M.; Kitts, D.D. Dietary Choline Intake: Current State of Knowledge Across the Life Cycle. Nutrients 2018, 10, 1513. [CrossRef] [PubMed]

23. USDA. The USDA National Nutrient Databank: Acquision, Evaluation \& Compilation of Food Composiion Data. Available online: https: / fdc.nal.usda.gov/ (accessed on 6 December 2021).

24. Derbyshire, E. Nutrition in the Childbearing Years; John Wiley and Sons: Hoboken, NJ, USA, 2011.

25. Lavery, A.M.; Brender, J.D.; Zhao, H.; Sweeney, A.; Felkner, M.; Suarez, L.; Canfield, M.A. Dietary intake of choline and neural tube defects in Mexican Americans. Birth Defects Res. A Clin. Mol. Teratol. 2014, 100, 463-471. [CrossRef]

26. Petersen, J.M.; Parker, S.E.; Crider, K.S.; Tinker, S.C.; Mitchell, A.A.; Werler, M.M. One-Carbon Cofactor Intake and Risk of Neural Tube Defects Among Women Who Meet Folic Acid Recommendations: A Multicenter Case-Control Study. Am. J. Epidemiol. 2019, 188, 1136-1143. [CrossRef] [PubMed]

27. Oyen, J.; Gjesdal, C.G.; Karlsson, T.; Svingen, G.F.; Tell, G.S.; Strand, E.; Drevon, C.A.; Vinknes, K.J.; Meyer, K.; Ueland, P.M.; et al. Dietary Choline Intake Is Directly Associated with Bone Mineral Density in the Hordaland Health Study. J. Nutr. 2017, 147, 572-578. [CrossRef]

28. Gao, X.; Wang, Y.; Randell, E.; Pedram, P.; Yi, Y.; Gulliver, W.; Sun, G. Higher Dietary Choline and Betaine Intakes Are Associated with Better Body Composition in the Adult Population of Newfoundland, Canada. PLoS ONE 2016, 11, e0155403. [CrossRef]

29. Lee, J.E.; Jacques, P.F.; Dougherty, L.; Selhub, J.; Giovannucci, E.; Zeisel, S.H.; Cho, E. Are dietary choline and betaine intakes determinants of total homocysteine concentration? Am. J. Clin. Nutr. 2010, 91, 1303-1310. [CrossRef] [PubMed]

30. Zeisel, S.H.; Mar, M.H.; Howe, J.C.; Holden, J.M. Concentrations of choline-containing compounds and betaine in common foods. J. Nutr. 2003, 133, 1302-1307. [CrossRef]

31. Chiuve, S.E.; Giovannucci, E.L.; Hankinson, S.E.; Zeisel, S.H.; Dougherty, L.W.; Willett, W.C.; Rimm, E.B. The association between betaine and choline intakes and the plasma concentrations of homocysteine in women. Am. J. Clin. Nutr. 2007, 86, 1073-1081. [CrossRef] [PubMed]

32. Fischer, L.M.; Scearce, J.A.; Mar, M.H.; Patel, J.R.; Blanchard, R.T.; Macintosh, B.A.; Busby, M.G.; Zeisel, S.H. Ad libitum choline intake in healthy individuals meets or exceeds the proposed adequate intake level. J. Nutr. 2005, 135, 826-829. [CrossRef] [PubMed]

33. Shaw, G.M.; Carmichael, S.L.; Yang, W.; Selvin, S.; Schaffer, D.M. Periconceptional dietary intake of choline and betaine and neural tube defects in offspring. Am. J. Epidemiol. 2004, 160, 102-109. [CrossRef] [PubMed]

34. Fawcett, K.; Sims, C.; Gaitliff, R. Associations Between Maternal Dietary Choline Intake During Pregnancy and Developmental Outcomes in Toddlers. Curr. Dev. Nutr. 2020, 4, 980. [CrossRef]

35. Moore, C.J.; Perreault, M.; Mottola, M.F.; Atkinson, S.A. Diet in Early Pregnancy: Focus on Folate, Vitamin B12, Vitamin D, and Choline. Can. J. Diet. Pract. Res. 2020, 81, 58-65. [CrossRef]

36. Zhu, J.; Liu, Y.H.; He, X.L.; Kohlmeier, M.; Zhou, L.L.; Shen, L.W.; Yi, X.X.; Tang, Q.Y.; Cai, W.; Wang, B. Dietary Choline Intake during Pregnancy and PEMT rs7946 Polymorphism on Risk of Preterm Birth: A Case-Control Study. Ann. Nutr. Metab. 2020, 76, 431-440. [CrossRef] [PubMed]

37. Pauwels, S.; Ghosh, M.; Duca, R.C.; Bekaert, B.; Freson, K.; Huybrechts, I.; Langie, S.A.S.; Koppen, G.; Devlieger, R.; Godderis, L. Maternal intake of methyl-group donors affects DNA methylation of metabolic genes in infants. Clin. Epigenet. $2017,9,16$. [CrossRef] [PubMed]

38. Groth, S.W.; Stewart, P.A.; Ossip, D.J.; Block, R.C.; Wixom, N.; Fernandez, I.D. Micronutrient Intake Is Inadequate for a Sample of Pregnant African-American Women. J. Acad. Nutr. Diet 2017, 117, 589-598. [CrossRef] [PubMed]

39. Masih, S.P.; Plumptre, L.; Ly, A.; Berger, H.; Lausman, A.Y.; Croxford, R.; Kim, Y.I.; O'Connor, D.L. Pregnant Canadian Women Achieve Recommended Intakes of One-Carbon Nutrients through Prenatal Supplementation but the Supplement Composition, Including Choline, Requires Reconsideration. J. Nutr. 2015, 145, 1824-1834. [CrossRef]

40. Goon, S.; Dey, S.R. A 24-hour dietary recall for assessing the intake pattern of choline among Bangladeshi pregnant women at their third trimester of pregnancy. Cent. Asian J. Glob. Health 2014, 3, 72. [CrossRef]

41. Lewis, E.D.; Subhan, F.B.; Bell, R.C.; McCargar, L.J.; Curtis, J.M.; Jacobs, R.L.; Field, C.J.; APrON team. Estimation of choline intake from $24 \mathrm{~h}$ dietary intake recalls and contribution of egg and milk consumption to intake among pregnant and lactating women in Alberta. Br. J. Nutr. 2014, 112, 112-121. [CrossRef] [PubMed]

42. Wu, B.T.; Dyer, R.A.; King, D.J.; Richardson, K.J.; Innis, S.M. Early second trimester maternal plasma choline and betaine are related to measures of early cognitive development in term infants. PLoS ONE 2012, 7, e43448. [CrossRef] [PubMed]

43. Villamor, E.; Rifas-Shiman, S.L.; Gillman, M.W.; Oken, E. Maternal intake of methyl-donor nutrients and child cognition at 3 years of age. Paediatr. Perinat. Epidemiol. 2012, 26, 328-335. [CrossRef]

44. EFSA. European Food Safety Authority. Use of the EFSA Comprehensive European Food Consumption Database in exposure assessment. EFSA J. 2011, 9, 2097.

45. Gossell-Williams, M.; Fletcher, H.; McFarlane-Anderson, N.; Jacob, A.; Patel, J.; Zeisel, S. Dietary intake of choline and plasma choline concentrations in pregnant women in Jamaica. West Indian Med. J. 2005, 54, 355-359. [CrossRef] 
46. Visentin, C.E.; Masih, S.; Plumptre, L.; Malysheva, O.; Nielsen, D.E.; Sohn, K.J.; Ly, A.; Lausman, A.Y.; Berger, H.; Croxford, R.; et al. Maternal Choline Status, but Not Fetal Genotype, Influences Cord Plasma Choline Metabolite Concentrations. J. Nutr. 2015, 145, 1491-1497. [CrossRef]

47. Fisher, M.C.; Zeisel, S.H.; Mar, M.H.; Sadler, T.W. Inhibitors of choline uptake and metabolism cause developmental abnormalities in neurulating mouse embryos. Teratology 2001, 64, 114-122. [CrossRef] [PubMed]

48. Fisher, M.C.; Zeisel, S.H.; Mar, M.H.; Sadler, T.W. Perturbations in choline metabolism cause neural tube defects in mouse embryos in vitro. FASEB J. 2002, 16, 619-621. [CrossRef]

49. Lewis, E.D.; Kosik, S.J.; Zhao, Y.Y.; Jacobs, R.L.; Curtis, J.M.; Field, C.J. Total choline and choline-containing moieties of commercially available pulses. Plant Foods Hum. Nutr. 2014, 69, 115-121. [CrossRef] [PubMed]

50. Lewis, E.D.; Zhao, Y.Y.; Richard, C.; Bruce, H.L.; Jacobs, R.L.; Field, C.J.; Curtis, J.M. Measurement of the abundance of choline and the distribution of choline-containing moieties in meat. Int. J. Food Sci. Nutr. 2015, 66, 743-748. [CrossRef] [PubMed]

51. Richard, C.; Lewis, E.; Zhao, Y.; Asomaning, J.; Jacobs, R.; Field, C.J.; Curtis, J. Measurement of the total choline content in 48 commercial dairy products or dairy alternatives. J. Food Compost. Anal. 2016, 2016, 1-8. [CrossRef]

52. Lecorguille, M.; Lioret, S.; de Lauzon-Guillain, B.; de Gavelle, E.; Forhan, A.; Mariotti, F.; Charles, M.A.; Heude, B. Association between Dietary Intake of One-Carbon Metabolism Nutrients in the Year before Pregnancy and Birth Anthropometry. Nutrients 2020, 12, 838. [CrossRef] [PubMed]

53. Radjursoga, M.; Lindqvist, H.M.; Pedersen, A.; Karlsson, B.G.; Malmodin, D.; Ellegard, L.; Winkvist, A. Nutritional Metabolomics: Postprandial Response of Meals Relating to Vegan, Lacto-Ovo Vegetarian, and Omnivore Diets. Nutrients 2018, $10,1063$. [CrossRef] [PubMed]

54. Perrin, M.T.; Pawlak, R.; Allen, L.H.; Hampel, D. Total Water-Soluble Choline Concentration Does Not Differ in Milk from Vegan, Vegetarian, and Nonvegetarian Lactating Women. J. Nutr. 2020, 150, 512-517. [CrossRef]

55. Wiedeman, A.M.; Whitfield, K.C.; March, K.M.; Chen, N.N.; Kroeun, H.; Sokhoing, L.; Sophonneary, P.; Dyer, R.A.; Xu, Z.; Kitts, D.D.; et al. Concentrations of Water-Soluble Forms of Choline in Human Milk from Lactating Women in Canada and Cambodia. Nutrients 2018, 10, 381. [CrossRef]

56. Wennberg, A.L.; Isaksson, U.; Sandstrom, H.; Lundqvist, A.; Hornell, A.; Hamberg, K. Swedish women's food habits during pregnancy up to six months post-partum: A longitudinal study. Sex Reprod. Healthc. 2016, 8, 31-36. [CrossRef] [PubMed]

57. Van der Pligt, P.; Olander, E.K.; Ball, K.; Crawford, D.; Hesketh, K.D.; Teychenne, M.; Campbell, K. Maternal dietary intake and physical activity habits during the postpartum period: Associations with clinician advice in a sample of Australian first time mothers. BMC Pregnancy Childbirth 2016, 16, 27. [CrossRef]

58. Harvard, T. Harvard Willett Food Frequency Questionnaire. Available online: https://snaped.fns.usda.gov/library/materials/ harvard-willett-food-frequency-questionnaire (accessed on 6 December 2021).

59. Carter, R.C.; Jacobson, S.W.; Booley, S.; Najaar, B.; Dodge, N.C.; Bechard, L.J.; Meintjes, E.M.; Molteno, C.D.; Duggan, C.P.; Jacobson, J.L.; et al. Development and validation of a quantitative choline food frequency questionnaire for use with drinking and non-drinking pregnant women in Cape Town, South Africa. Nutr. J. 2018, 17, 108. [CrossRef]

60. Ortega, R.M.; Perez-Rodrigo, C.; Lopez-Sobaler, A.M. Dietary assessment methods: Dietary records. Nutr. Hosp. 2015,31 (Suppl. 3), 38-45. [CrossRef] [PubMed]

61. Shaneshin, M.; Jessri, M.; Rashidkhani, B. Validity of energy intake reports in relation to dietary patterns. J. Health Popul. Nutr. 2014, 32, 36-45. [PubMed]

62. Wiedeman, A.M.; Dyer, R.A.; Innis, S.M. Variability in Plasma Free Choline and its Relation with Diet and Potential Plasma Biomarkers. FASEB J. 2015, 29, 919-922. [CrossRef]

63. Romano, K.A.; Martinez-Del Campo, A.; Kasahara, K.; Chittim, C.L.; Vivas, E.I.; Amador-Noguez, D.; Balskus, E.P.; Rey, F.E. Metabolic, Epigenetic, and Transgenerational Effects of Gut Bacterial Choline Consumption. Cell Host Microbe 2017, 22, 279-290. [CrossRef] [PubMed]

64. Goh, Y.Q.; Cheam, G.; Wang, Y. Understanding Choline Bioavailability and Utilization: First Step Toward Personalizing Choline Nutrition. J. Agric. Food Chem. 2021, 69, 37. [CrossRef]

65. Schwarzenberg, S.J.; Georgieff, M. Advocacy for Improving Nutrition in the First 1000 Days to Support Childhood Development and Adult Health. Committee on Nutrition. Paediatrics 2018, 141, e20173716. [CrossRef] 